Ebisu Ebisu

Études japonaises Études japonaises

47 | printemps-été 2012

Catastrophes du 11 mars 2011, désastre de

Fukushima : fractures et émergences

\title{
Un modèle géographique de la catastrophe
}

A Geographical Model Regarding Disasters

地理的焱害モデル

\section{Marie Augendre}

\section{(2) OpenEdition}

Journals

Édition électronique

URL : http://journals.openedition.org/ebisu/219

DOI : 10.4000/ebisu.219

ISSN : 2189-1893

Éditeur :

Institut français de recherche sur le Japon (UMIFRE 19 MAEE-CNRS), Maison franco-japonaise

Édition imprimée

Date de publication : 1 juin 2012

Pagination : 27-38

ISSN : 1340-3656

Référence électronique

Marie Augendre, "Un modèle géographique de la catastrophe », Ebisu [En ligne], 47 | printemps-été 2012, mis en ligne le 03 avril 2014, consulté le 01 mai 2019. URL : http://journals.openedition.org/ ebisu/219; DOl : 10.4000/ebisu.219

(c) Institut français de recherche sur le Japon à la Maison franco-japonaise 


\title{
Un modèle géographique de la catastrophe
}

\author{
Marie AUGENDRE
}

"Il n'y a pas de soleil sans ombre, et il faut connaître la nuit. " Albert Camus,

Le mythe de Sisyphe (1942).

Les événements qui se sont enchaînés au Japon ont donné naissance le 11 mars 2011 à une catastrophe hybride, amorcée par un double aléa naturel et amplifiée par un accident nucléaire, où s'emboîtent les échelles de temps et d'espace, où se combinent effets proximaux et répercussions lointaines, temporalités instantanées et à long, voire très long terme. La notion d'impermanence des choses et des êtres vivants, mujō 無常, deviendrait alors un refuge consolant, face à une manifestation localement extrême de la nature et une contamination radioactive durable et étendue.

$\mathrm{Au}$ Japon, l'ordre et les aléas naturels sont connus de longue date et pris en charge par les chercheurs, par le pouvoir politique ou encore dans les communautés locales. Les risques résultants sont surveillés et évalués; ils font l'objet de mesures de prévention et de protection. Les acteurs de la société ont appris à vivre avec ces risques, en les atténuant ou en s'en accommodant, à assimiler les impacts des catastrophes, à résorber leurs effets négatifs. Cette coexistence, kyōson 共存, butte sur le caractère inédit du désastre de 2011, qui souligne la résilience du pays autant qu'il montre ses limites.

Marie Augendre (marie.augendre[at]univ-lyon2.fr) est géographe, maîtresse de conférences à l'université Lyon 2 et membre d'EVS (UMR 5600). Sa thèse (2008) porte sur la coexistence avec le risque volcanique au Japon. 
En effet, l'archipel à la fois exposé à une nature brutale (typhons, séismes, tsunamis, éruptions volcaniques) et confronté à des mécanismes politicoindustriels effrayants (secret, déni, falsification), a aussi été capable de supporter les chocs de toute nature, de se reconstruire pour perdurer, en tirant profit des profusions du milieu, de la cohésion sociale, de l'innovation et de son déploiement. Cette fois, la crise est loin d'être achevée, et le Japon voit l'intégrité d'une partie de son territoire remise en question.

Le risque perdure tout en se transformant, récalcitrant à toutes les mesures prises pour l'atténuer et s'en prémunir (November et al. 2011 : 33). Cette coévolution qui unit risque et territoire est la transposition du processus que le biologiste Claude Combes définit comme l'adaptation incessante d'adversaires pour ne pas se laisser distancer, dans " un enchaînement de pressions sélectives réciproques " (Combes 2001 : 222). Avant d'illustrer ses manifestations, il convient de présenter l'organisation temporelle et spatiale de toute catastrophe. Le cycle sismique offre justement un modèle transposable.

\section{Tremblement de terre, séisme social : un modèle analogique}

Un tremblement de terre, jishin 地震, est une secousse ou une série de secousses affectant brutalement l'écorce terrestre. Il ne survient pas n'importe où et n'importe quand, bien qu'il ne soit toujours pas prévisible de manière utile à l'alerte des populations menacées. Le géophysicien américain Harry Fielding Reid (1859-1944) est le premier à avoir exprimé, suite au séisme de 1906 à San Francisco, la théorie du rebond élastique pour expliquer la genèse et la répétition des tremblements de terre à un endroit donné (Madariaga \& Perrier 1991 : 80). Cette théorie, formulée un demisiècle avant que la tectonique des plaques ne vienne rendre compte des mouvements de la surface du globe, a depuis été intégrée dans la notion de cycle sismique. Alors qu'en profondeur les lents déplacements internes au manteau ductile provoquent l'accumulation de contraintes dans la croûte rigide, celle-ci reste bloquée jusqu'à ce que les forces de friction dépassent le seuil de résistance de la roche. L'énergie est alors brutalement libérée, essentiellement sous forme d'ondes et d'un "rebond ", déplacement des compartiments le long d'une faille pour rattraper le retard accumulé. Une 
partie de ce mouvement s'effectue après la rupture principale, sous forme de nouveaux séismes (yoshin 余震, répliques, ou yūhatsu jishin 誘発地震 séismes provoqués). Les milieux traversés par les ondes sont à l'origine d'effets de site (amplification des secousses, liquéfaction du sol) et d'effets induits (tsunami, glissement de terrain, effondrements). Si le séisme relâche les contraintes accumulées localement, il conduit aussi à les abaisser ou au contraire à les augmenter sur plusieurs dizaines de kilomètres alentours. Ainsi, il accélère ou au contraire ralentit la survenue de séismes ultérieurs dans une même région. Ces effets, qui traduisent un réajustement spatial de la contrainte de rupture de Coulomb (King et al. 1994), peuvent survenir quelques minutes ou plusieurs décennies après le séisme initial, et multiplier par dix la probabilité d'un séisme sur une faille voisine (Toda et al. 2011).

En résumé, un cycle sismique est composé de quatre phases (Philip et al. $2007: 25$ sqq.) :

- une lente mise en tension de la faille ;

- l'occurrence de précurseurs (phase discutée et mal connue, le plus souvent identifiée a posteriori, et seulement pour certains séismes) ;

- la rupture sismique au niveau du foyer ;

- la propagation des ondes jusqu'en surface et des ruptures en chaine le long d'un plan de faille ou sur des failles voisines, s'accompagnant de processus en relais, d'effets induits directs et indirects, qui s'atténuent globalement avec le temps et la distance ;

- la reprise de la mise en charge, qui correspond au retour à la phase initiale du cycle.

La catastrophe du 11 mars se décrypte selon de semblables étapes. Toute catastrophe naturelle ou technologique d'ailleurs suit un cycle similaire à celui d'un tremblement de terre. Pourtant, si les séismes de grande magnitude se répètent inexorablement en réponse au mouvement des plaques, à un rythme géologique difficile à appréhender par le temps des instruments ou celui de la vie humaine, un désastre n'a rien d'inéluctable pour une société ; il peut ne pas avoir lieu... Tout comme certaines failles ne sont pas systématiquement bloquées, mais peuvent connaitre des glissements asismiques (fluage ou creep), parfois qualifiés de "séismes lents " (Hirose et al. 1999). 
La mise en tension de la catastrophe correspond à ce qui la prépare en profondeur : de la position et la configuration de la côte du Sanriku, avec ses rias qui font face à une fosse de subduction active, aux choix énergétiques du Japon, qui s'est tourné vers le nucléaire civil et a implanté une cinquantaine de réacteurs à proximité de la mer pour le refroidissement, en passant par les caractéristiques socio-économiques et démographiques du Tōhoku, qui expliquent que Tokyo, bien qu'épargnée par la secousse, ait subi des contrecoups liés aux flux pendulaires et à son approvisionnement en électricité ou en denrées alimentaires.

La mise en tension intègre aussi le jeu de la mémoire et de l'oubli des événements passés, ainsi que les représentations du risque invalidées par les événements de mars. Ainsi, la cartographie nationale de l'aléa sismique (HERP 2005) est focalisée par la région du Tōkai et néglige des périphéries de la mégalopole où se répètent pourtant des séismes majeurs depuis deux décennies au moins (Toda \& Awata 2007 ; Geller 2011). Ni les tsunami kinenhi 津波記念碑 (ou tsunami sekihi 津波石碑) ${ }^{1}$, stèles commémoratives qui rappellent les vagues historiques et mettent silencieusement en garde contre les constructions à faible altitude, ni les bōsai kunren 防災訓練, exercices d'évacuation régulièrement mis en scène dans tout le pays, n'ont suff, pas plus que le système d'alerte précoce, les plans de prévention, les briselames, bōhatei 防波堤, ou les digues côtières bōchōtei 防潮堤.

Les précurseurs, non identifiés comme tels à l'avance sauf par des Cassandres (Mogi 2004), sont les incidents et catastrophes du passé, comme le tsunami de 869 (Minoura et al. 2001) ou l'accident de la centrale nucléaire de Kashiwazaki-Kariwa, suite au séisme de Niigata en 2007 (CNIC 2007).

La rupture commence le 11 mars par un séisme qui déclenche un tsunami, qui s'accompagnent de liquéfactions et d'inondations marines et causent près de 16000 victimes, sans compter plus de 3000 disparus (Keisatsuchō 2011). Rapporté à la population communale, les taux de décès atteignent localement 5 à $11 \%$ (Isoda 2011a). Les dégâts matériels colossaux sont impossibles à estimer précisément en raison du coût non établi de la décontamination. Le gouvernement indiquait d'ores et déjà

1. Elles sont recensées, localisées et parfois photographiées dans cette base de données en ligne : <http://protea.dbms.cs.gunma-u.ac.jp/>. 
17 milliards de yens (160 millions d'euros) en juin 2011. Des quartiers entiers sont arasés dans des villages portuaires, notamment sur le littoral des trois départements d'Iwate, de Miyagi et de Fukushima, où plus de 330000 résidences sont à demi ou totalement détruites. Cette rupture se répercute instantanément sur l'essentiel du trafic routier, ferroviaire et aérien du Tōhoku, puis dans les instants et les jours qui suivent, se poursuit par des incendies, un accident nucléaire et près de 600 répliques de magnitude supérieure à cinq (Kishōchō 2011).

L'expansion de la catastrophe a atteint une ampleur mondiale, puisque par effets dominos elle a conduit la Suisse à sortir progressivement du nucléaire civil, puis a coupé court aux tergiversations de l'Allemagne (gouvernement Schroeder en 2000) et de l'Italie (référendum de 1987) en la matière. Le consensus nucléaire est ébranlé même en France, où une campagne de tests de sûreté et une réévaluation des coûts de démantèlement sont conduites.

La place modeste du Tōhoku dans l'économie nationale (moins de 7 \% du PNB), même si cette région héberge des fournisseurs capables d'enrayer les chaînes de production informatique ou automobile au-delà de l'archipel, l'ampleur des destructions matérielles et la question pendante de la radioactivité font que la reprise qui avait caractérisé Tokyo en 1923 ou Kobe en 1995 nécessitera plus d'efforts. Localement, la catastrophe a modifié le paysage littoral pour longtemps, bien que les photographies de presse publiées six mois après l'événement attestent de la rapidité avec laquelle les populations locales reprennent pied $^{2}$. Elle a relancé le secteur de la construction mais a atteint durablement nombre de petits commerces, qui constituaient l'ossature économique des communes littorales, et fragilisé les activités halieutiques ; elle aura des effets sur la santé des populations qui ont été, sont et seront encore pendant des décennies au contact des radionucléides, sans qu'il soit possible encore d'évaluer la gravité des séquelles et l'ampleur de la population touchée. Les quelques 25 millions de tonnes de débris, gareki 瓦砂, qu'il faut déblayer, trier, évacuer ou éliminer, ont commencé une vie indépendante et constituent des stocks et des flux à gérer, avec des précautions particulières lorsqu'ils sont polluants ou irradiés.

2. Sur le site du quotidien californien Sacramento Bee : <http://blogs.sacbee.com/ photos/2011/09/japan-marks-6-months-since-ear.html>. 
La subsidence a atteint $75 \mathrm{~cm}$ par endroits, comme à Ishinomaki (HERP, 2011), ce qui contribue à la remise en charge du système en fragilisant un peu plus les sites côtiers. La reconstruction correspond à l'effacement de la catastrophe passée autant qu' elle tisse les conditions d'une remise en tension du risque, qui inclut désormais un danger latent, invisible et controversé.

\section{Un révélateur de la coévolution entre risque et société}

La reconstruction a commencé sans tarder dans le Tōhoku. Elle est fondée sur des solutions déjà éprouvées lors de catastrophes naturelles de moindre ampleur, mais ayant pareillement provoqué des destructions matérielles, imposé de reloger les populations affectées pendant des mois voire des années, puis conduit à augmenter le niveau de protection des quartiers rebâtis. Ainsi, la mise à disposition de logements sociaux et la construction rapide de maisons temporaires préfabriquées est un invariant des secteurs affectés par les séismes, comme à Kobe (1995) ou à Niigata (2004 puis 2007). Elle est aussi mise en œuvre après des éruptions volcaniques, notamment à Miyake-jima, île de près de 4000 habitants qui a été évacuée de tous ses résidents habituels de la fin de l'été 2000 jusqu'en février 2005 (Perrin $2008: 37)$.

La reconstruction sur des terrains surélevés, comme le prévoit la ville d'Onagawa ${ }^{3}$, ou gagnés sur la mer, qui joint l'utile de trouver des terrains publics disponibles à la nécessité de se débarrasser des montagnes de débris abandonnés par les vagues en mars 2011, n’est pas non plus une innovation. Suite à la dernière éruption de l'Unzen 雲仙 (1991-1995), Shimabara 島原 avait ainsi créé deux nouveaux quartiers sur les dépôts de lahars, le triangle d'Annaka (Annaka sankaku 安中三角) qui surplombe deux bras d'un chenal torrentiel, et le terre-plein du quartier de Heisei (Heisei-chō 平成町) à son delta (Augendre 2008 : 52 sqq.).

Mobiliser le secteur de la construction pour remettre en état les infrastructures, développer ou renforcer les ouvrages de protection, sont deux des fondements de l'aménagement du territoire qui ont valu au Japon le

3. Le plan de reconstruction est présenté sur le site internet de la ville : <http://www. town.onagawa.miyagi.jp/hukkou/keikaku.html>. 
sobriquet d'État-BTP, doken kokka 土建国家, et constituent un véritable syndrome de la pelle mécanique.

Les ouvrages de " protection contre les sables ", sabō 砂防, sont depuis plus d'un siècle mis à contribution sur les torrents et rivières, dans les bassins de réception et les chenaux torrentiels, sur les pentes affectées de glissements de terrain et en périphérie des volcans actifs. Les tétrapodes en béton, dont les plus gros pèsent 80 tonnes et font six mètres d'envergure ${ }^{4}$, sont des composants familiers et symboliques des paysages littoraux, où ils jalonnent le trait de côte, constituent des enrochements au large ou des épis, et ourlent quais et jetées. Ils partagent avec les autres sabō une triple logique spatiale : celle de confiner des flux potentiellement destructeurs, sédiments, eau salée ou polluée, hors de l'espace habité, celle de transférer des flux financiers du centre métropolitain vers des périphéries en "contraction " démographique et économique, et celle enfin de matérialiser dans le paysage une frontière symbolique entre l'espace social, contrôlé par l'État, et l'espace sauvage qui apporte bienfaits ou destruction. Tarō 田老 et Kamaishi 鉒石, deux ports du département d'Iwate, étaient dotés de digues de grande ampleur, destinées à les protéger des tsunamis récurrents. Sources de fierté et génératrices d'un sentiment de sécurité, elles n’ont pas résisté aux effets conjoints de la liquéfaction induite par le séisme (Aydan \& Ōta 2010) et de l'ampleur du tsunami. Empêchant le reflux, elles ont même contribué à prolonger l'inondation des terres côtières. En bref, si les mesures de prévention et de protection prises ont pour l'essentiel joué leur rôle, elles ont localement eu des effets non désirés ou imprévus et provoqué des rétroactions négatives.

Cette logique de séparation et de confinement se retrouve dans les consignes collectives qui intiment aux résidents de la zone entre 20 et $30 \mathrm{~km}$ des réacteurs de Fukushima de se calfeutrer chez eux, ainsi que dans les seuils de radioactivité définissant des concentrations acceptables. Impossibles à systématiser, ces précautions sont tout autant inapplicables que le seraient, notamment en raison de l'attrait supérieur des richesses halieutiques, des mesures visant à supprimer le peuplement du littoral afin de séparer les populations des dangers qui les menacent, comme les bâtisseurs des sanctuaires anciens toujours en place à quelques mètres de la côte atteinte par la mer le 11 mars en avaient pourtant compris la nécessité.

4. Voir : <http://www.fudotetra.co.jp/products/tetrapod.html>. 
Inversement, le mot d'ordre traditionnel des communautés de pêcheurs du Sanriku, plusieurs fois frappées par des tsunamis au $\mathrm{xx}^{\mathrm{e}}$ siècle, rappelle la fragilité humaine et fait passer la préservation du collectif par le salut individuel. "Sauve qui peut " ou " chacun pour soi " pourraient traduire l'expression dialectale inochi (ou parfois tsunami) tenden-ko 命(津波) んでんこ, principe selon lequel prendre les autres en charge, y compris sa famille, ou se préoccuper de biens matériels au lieu de courir sans délai vers un point haut est risquer sa perte en cas de tsunami. Ce précepte signifie aussi qu'il est vain pour un survivant de se sentir coupable de n'avoir pu sauver personne (Sekine 2011). Cette conduite est moins facile à mettre en ouvre aujourd'hui qu'en 1896 ou en 1933, en raison du vieillissement de la population. En effet, les plus âgés requièrent une assistance pour se déplacer, et les limites de ce sauve qui peut se lisent dans les caractéristiques démographiques des victimes, dont les plus de 75 ans représentent les deux tiers, soit le double de leur part dans la population locale (Isoda, 2011b).

Ces ajustements et ces rééquilibrages de contraintes matérielles, sociales et culturelles témoignent de la coévolution entre risque et société. La gestion du risque sismique par les individus et les collectifs, institutionnels ou non, auxquels ils appartiennent, a contribué à élaborer des représentations et des dispositifs permettant de limiter les pertes causées par le tsunami, comparativement au bilan du séisme de Sumatra (2004), de magnitude pourtant similaire. Toutefois, ces mesures ont leur limite, que tendent à masquer le discours suffisant qui les accompagne et la confiance en la technique. En réalité elles ne peuvent prétendre maîtriser tous les effets destructeurs des aléas, qu'ils soient naturels ou technologiques. Ainsi, en promouvant et en acceptant le nucléaire civil, le risque d'accident et la question non résolue de ses déchets en dépit du danger qu'ils représentent, cette gestion probabiliste a débouché sur des dommages matériels, sanitaires et environnementaux irréparables, latents voire héréditaires, en tout cas de long terme.

Appréhender l'événement catastrophique à travers le filtre d'un modèle qui intègre les temporalités et les échelles spatiales permet tout d'abord de révéler ses causes profondes par la prise en compte de sa mise en tension. Elle amène à intégrer les héritages et les dépendances, les rapports de force, les flux financiers, les choix politiques, et des territoires plus vastes que ceux qui sont touchés, sans se limiter à des déclencheurs directs. Intégrer 
ensuite l'ensemble des effets et répercussions de la catastrophe qui constituent son déploiement, permet de dépasser les données individuelles, locales et immédiates.

Comme toute forme d'appréhension modélisatrice du réel, ce prisme a aussi ses limites et ses biais. En privilégiant les facteurs conduisant à la catastrophe, il amène notamment à laisser de côté toute une économie de la prévention qui est bien plus malaisée à évaluer. Il tend également à oblitérer la situation des hommes et des femmes qui sont aux prises avec le quotidien de la catastrophe, leurs voix, leurs choix et leurs parcours individuels. Tous contribuent de fait à une reconfiguration qui concourt à dessiner de nouveaux territoires, de nouveaux niveaux d'intervention, de nouvelles formes de responsabilité et de nouvelles manières de se protéger. La contamination d'une partie du territoire terrestre et marin pourrait bien délimiter un nouveau front pionnier, sinon dans l'immédiat accroître la marginalisation des régions touchées et faire rétrécir l'écoumène.

8 mars 2012

Remerciements :

Je remercie très chaleureusement les relecteurs pour leur patience et leur lecture critique, ainsi que mes collègues Isabelle Lefort et Alain Le Griel, pour leur contribution à la formalisation des idées présentées ici. 


\section{Bibliographie}

NB : sauf indication contraire, le dernier accès aux liens date du 8 mars 2012.

AUGENDRE Marie, 2008

Kazan to tomo ni ikiru 火山と共に生き

る. Vivre avec le volcan : une géographie du risque volcanique au Japon, thèse de doctorat, sous la direction de Philippe PELLETIER, université Lumière Lyon 2. En ligne: <http://demeter.univ-lyon2.fr/ sdx/theses/lyon2/2008/augendre_m>.

AYDAN Ömer \& ŌTA Yoshimi, 2010 Shaking Table Tests on Settlement of Breakwaters Made of Stone Block on Liquefiable Ground, Tōkai University, Dept. of Marine Civil Eng., rapport non publié.

COMBES Claude, 2001 [réed. 2010] Les associations du vivant : l'art d'être parasite, Paris, Flammarion.

CNIC, 2007

Kashiwazaki-Kariwa Earthquake. Japan's Nuclear Safety Shaken to the Roots. En ligne : <http://cnic.jp/english/ newsletter/nit119/nit119articles/ kkearthquake.html>.

GELLER Robert J., 2011

"Shake-Up Time for Japanese

Seismology », Nature, 472 : 407-409.

HERP Jishin chōsa kenkyū suishin honbu 地震調査研究推進本部 (Comité central pour la promotion de la sismologie), 2005 Jishindō yosoku chizu 地震動予測地図
(Carte de prévision de l'activité sismique). En ligne : <http://www.jishin. go.jp/main/chousa/09_yosokuchizu/ honpen.pdf $>$.

HERP, 2011

Hendō bekutoru zu (jōge) 変動ベクトル図 （上下) (Carte vectorielle des mouvements verticaux). En ligne: $<$ http://www.jishin.go.jp/main/ chousa/11mar_sanriku-oki2/p06.htm>.

HIROSE Hitoshi, HIRAHARA Kazurō, KIMATA Fumiaki et al., 1999 «A Slow Thrust Slip Event Following the Two 1996 Hyuganada Earthquakes Beneath the Bungo Channel, Southwest Japan ", Geophysical Research Letters, 26: 3237-3240.

ISODA Yuzuru, 2011a «The Impact of Casualties of $+20,000$ :

Deaths and Missing Persons by Municipalities », The 2011 East Japan Earthquake Bulletin of the Töhoku Geographical Association. En ligne : $<$ http://wwwsoc.nii.ac.jp/tga/disaster/ articles/e-contents1.html>.

\section{ISODA Yuzuru, 2011b}

« God's Taste Victims' Attributes Based on the List of Victims ", The 2011 East Japan Earthquake Bulletin of the Tōhoku Geographical Association. En ligne : $<$ http://wwwsoc.nii.ac.jp/tga/disaster/ articles/e-contents3.html>. 
KING Geoffrey C.P., STEIN Ross S. et LIN Jian, 1994

"Static Stress Changes and the

Triggering of Earthquakes », Bulletin of the Seismological Society of America, 84 : 935-953.

MADARIAGA Raul \& PERRIER Guy, 1991 Les tremblements de terre, Paris, Éditions du CNRS.

\section{MINOURA K., IMAMURA F., SUGAWARA \\ D. et al., 2001}

«The 869 Jōgan Tsunami Deposit and Recurrence Interval of Largescale Tsunami on the Pacific Coast of Northeast Japan », Journal of Natural Disaster Science, 23 (2) : 83-88.

MOGI Kiyō, 2004

«Two Grave Issues Concerning the Expected Tokai Earthquake », Earth Planets Space, 56 : LI-LXVI.

\section{MORIN Edgar, 1990}

Introduction à la pensée complexe, Paris, éd. ESF.

\section{NOVEMBER Valérie, VIOT Pascal} et PENELAS Marion (eds.), 2011 Habiter les territoires à risque, Lausanne, Presses polytechniques et universitaires romandes, Coll. Espace en société.

PERRIN Julie, 2008

Retour à Miyake-jima ou Vivre avec l'île-volcan. Réappropriations et représentations, Mémoire de Master 1 sous la direction de Philippe PELLETIER, département de géographie, université Lumière Lyon 2.
PHILIP Hervé, BOUSQUET Jean-Claude et MASSON Frédéric, 2007

Séismes et risques sismiques, Paris, Dunod.

Keisatsuchō 警察庁 (Agence de la police nationale - NPA), 2011

Situation du nombre de victimes au 12 décembre 2011 : <http://www.npa. go.jp/archive/keibi/biki/higaijokyo.pdf>.

Kishōchō 気象庁 (Agence météorologique - JMA), 2011 Nombre de répliques au 8 décembre 2011 : <http://www.jma.go.jp/jma/ en/2011_Earthquake.html>.

SEKINE Ryōhei, 2011

« Did the People Practice "Tsunami Tendenko"? », The 2011 East Japan Earthquake Bulletin of the Tōhoku Geographical Association. En ligne: <http://wwwsoc.nii.ac.jp/tga/disaster/ articles/e-contents22.html>.

TODA Shinji \& AWATA Yasuo, 2008 « Does the 2007 Noto Hanto Earthquake Reveal a Weakness in the Japanese National Seismic Hazard Map that Could Be Remedied with Geological Data? ", Earth Planets Space, 60 : 1047-1052.

TODA Shinji, LIN Jian et STEIN Ross S., 2011 « Using the $2011 \mathrm{M}=9.0$ Tohoku Earthquake to Test the Coulomb Stress Triggering Hypothesis and to Calculate Faults Brought Closer to Failure », Earth Planets Space, 63 : 1-6. 


\section{USGS, 2011}

Description des séismes du 9 et du 11 mars 2011 : <http://earthquake. usgs.gov/earthquakes/recenteqsww/ Quakes/usb0001r57.php> et <http:// earthquake.usgs.gov/earthquakes/ recenteqsww/Quakes/usc0001xgp. php>.

\section{Yomiuri shinbun, 2011}

« $92.5 \%$ of Disaster Victims Drowned, NPA Finds », Daily Yomiuri Online :

<http://www.yomiuri.co.jp/dy/national/ T110420005996.htm>. 